\title{
Detection and classification of leaf diseases using integrated approach of support vector machine and particle swarm optimization
}

\author{
Prabhjeet Kaur*, Sanjay Singla, Sukhdeep Singh \\ Computer Science Engineering, IET Bhaddal Technical Campus, Rupnagar, India
}

\section{A RT ICLE INFO}

\section{Article history:}

Received 19 March 2017

Received in revised form

2 July 2017

Accepted 7 July 2017

\section{Keywords:}

Leaf disease detection

Particle swarm optimization

K-means clustering

Support vector machine

\begin{abstract}
A B S T R A C T
Plant diseases are one of the common factors responsible for the decrease in plant growth. Plant diseases are analyzed with their leaves. Many researchers have analyzed the different methods to detect the leaf diseases but the evaluated results are not appropriate enough. So, in this paper we have presented an integrated approach of particle swarm optimization (PSO) and support vector machine (SVM) for plant leaf disease detection and classification. Here, the disease affected dataset of plant leaves is considered that is suffered with four diseases Cercospora leaf spot, bacterial blight, anthracnose, and Alternaria alternata. The main objective of this paper is to detect the disease affected portion of leaf and healthy portion of leaf. We have calculated the percentage of leaf affected portion with their classification. Overall results are evaluated in the form of accuracy of proposed integrated approach.
\end{abstract}

(C) 2017 The Authors. Published by IASE. This is an open access article under the CC BY-NC-ND license (http://creativecommons.org/licenses/by-nc-nd/4.0/).

\section{Introduction}

It has been found that there is the existence of more than 1.7 million living species on the earth surface. Out of this huge number of living algae, animals, plants and humans; only plants are the species that are essential to make life cycle for the human life (De Groot et al., 2002). Plants provide a way of living to humans in the form of breathing oxygen to other essential resources. A plenty of medicines and food bases are the gift of plants to humans. So, plants are essential components of human life and needed to be protected at each stage (Raven et al., 2005).

In plants, major useful species is known in the form of agriculture crops. These crops are the feed for more than $70 \%$ of our country population. But the production of crops can be affected by diseases and these diseases are not visible with naked eyes. Plant diseases are analyzed from the affected leaf portion (Sankaran et al., 2010). One method to detect these diseases is manually detection with the help of some botanic expert but manually detection of leaf diseases is much laborious and time consuming task.

So, there is the need of some autonomous method to detect the plant diseases with more efficiency as

\footnotetext{
* Corresponding Author.

Email Address: prabhbajwa05@gmail.com (P. Kaur) https://doi.org/10.21833/ijaas.2017.08.012

2313-626X/@ 2017 The Authors. Published by IASE.

This is an open access article under the CC BY-NC-ND license

(http://creativecommons.org/licenses/by-nc-nd/4.0/)
}

compare to manual detection. In this paper, we are using integrated approach of SVM and PSO for plant leaf disease detection.

Plants disease detection involves basically five steps: Image Acquisition, Image Pre-processing, Image Segmentation, Feature Extraction and Leaf Disease detection and classification (Khirade and Patil, 2015). In this proposed integrated concept, initially image is converted from RGB to HSV color, then image contrast enhanced, Image segmented using k-means clustering, further SVM and PSO are applied for feature extraction and disease detection. After disease detection, we have also evaluated the disease affected portion of leaf with their percentage value. Overall results are evaluated with the help of Accuracy with maximum possible iterations.

Other sections of the paper are described as: Section 2 discussed about the work related to plant leaf disease detection, Section 3 presents the considered basic concepts for proposed approach, Section 4 presents the considered dataset, Section 5 discuses about proposed concept, Section 6 shows the calculated results based on the considered parameters and Section VII concludes the paper.

\section{Literature survey}

There is the existence of the list of methods used by researchers for the detection of plant leaf diseases.

Patil and Bodhe (2011) measured the disease severity in the sugar cane with the help of digital 
image processing. They also developed an image processing and fuzzy logic based disease grading method. In this method total leaf area and total disease area were calculated. Atas et al. (2011) have investigated and detected aflatoxin contaminated chili pepper with the help of Halogen illuminations and UV. Authors have proposed complete differentiation of successive spectral energy features. Contreras et al. (2012) have premeditated about the elegant sensor competent of providing that robust, exact and accurate quantification of worldwide indication in leaves of diseased plants. The authors used FPGA support smart sensor capable to perform nondestructive, real-time analysis of infected and healthy leaf images to quantify multiple symptoms.

Wang et al. (2012) have developed a technique to recognize digital image and pattern detection of plant infection. In this method various neural network methods are used, i.e. radial basis function (RBF) neural networks, back-propagation (BP) networks, probabilistic neural networks (PNNs) and generalized regression networks (GRNNs) to differentiate between rust from wheat leaf and to discriminate downy mildew infection from powdery mildew infection. Barbedo (2013) have discussed about the pathogens in cultivated area. The result indicated the yield losses in field crop by pathogens and represents less income to the farmers due to the inferior product superiority as well as higher prices to the consumer due to the lesser offering of goods. Gavhale et al. (2014) have performed the process of image processing for detection of unhealthy region of citrus leaf. Citrus diseases are like citrus canker, anthracnose, overwatering and citrus greening disease. To achieve the type of final disease type, authors have used k-means clustering for the segmentation of image, GLCM for texture feature extraction, means of mean for color feature extraction and SVM for the final classification of citrus disease.

Pujari et al. (2014) has presented the work for the identification and classification of horticulture/agriculture crops affected from fungal diseases. Authors have used different image processing techniques for the identification of fungal affected different crops like commercial crops, fruit crops, vegetable crops and cereal crops. Singh and Misra (2015) has presented the existing work for the detection of unhealthy region of plant leaves. Authors have described the framework for the detection and classification of plat leaf diseases. Dandawate and Kokare (2015) have used support vector machine concept for the detection and classification of soybean plants as diseased or healthy species. Authors have used the SIFT approach that automatically recognizes plant species by their leaf shape. The proposed concept for soybean plant diseases shows an average accuracy of 93.79\%. Rothe and Kshirsagar (2015) have proposed pattern recognition techniques for the detection and classification of cotton leaf diseases of Alternarnia, Myrothecium and bacterial blight.

\section{Basic concepts}

This section describes the basics of Support Vector Machine and Particle Swarm Optimization.

\subsection{Support vector machine}

Support Vector Machine (SVM) Suykens and Vandewalle (1999) is one of the best-known and significant supervised statistical learning methods for the solution of classification and regression problems with strong theoretical foundations based on the principle of structural risk minimization. The SVM, proper selection of parameters is most important. However, improper selecting of SVM parameters usually leads to very poor generalization capabilities. Searching the optimal SVM parameters is decisive for achieving exceptional performance (Tong and Chang, 2001).

Support Vector machine works on the three steps based mechanism. First step is to take input data in a training phase, second step is to build a model using the input data and final step is output with a hypothesis that can predict the function with future data (Amari and Wu, 1999). The goal of SVM is to produce a model which predicts target value of data instances in the testing set which are given only the attributes.

\subsection{Particle swarm optimization}

Particle Swarm Optimization (PSO) is a nature inspired technique that came in existence by the optimization behaviour and sharing behaviour of the population of the flying birds is proposed by Kennedy (2011). In Particle Swarm Optimization, particles are considered as the flock of birds that are the main agents of the optimization techniques. The two main optimization factors of this technique are local search and global search optimization features. In local search, particles get their own individual best optimized solution using their own experiences. In global search, the experience of one bird is shared with the experience of another bird and finally gets result as a global best solution (Clerc, 2010).

This algorithm works in an iteration manner and moves closer to the best solution. Initially particles begin the process by the casual fly in the form of population of $N$ particle solution. In the Sdimensional space, the position of the $i$ th particle is represented as a point in this space where $S$ is the number of variables participated (Poli et al., 2007). In the entire process, particles try to find the global best solution.

\section{Dataset considered}

The proposed integrated concept is used for the detection of plant leaf diseases. For this, we have considered the expert dataset having leaf of four different types of diseases (Cercospora leaf spot, bacterial blight, anthracnose, and Alternaria 
alternata). Some sample images for the different
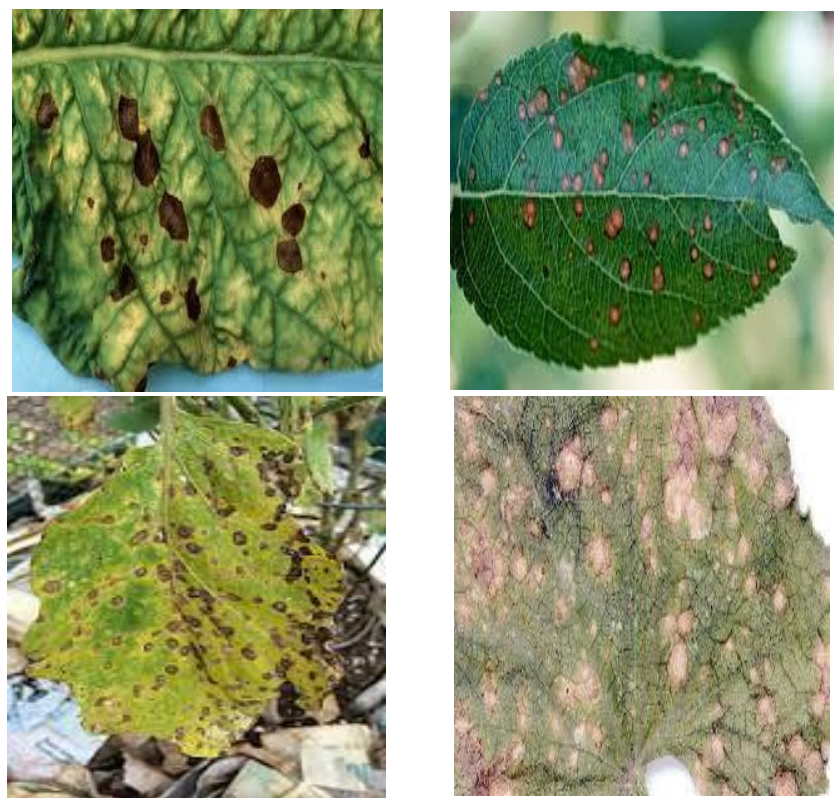

Fig. 1: Considered expert dataset diseases are shown as Fig. 1.
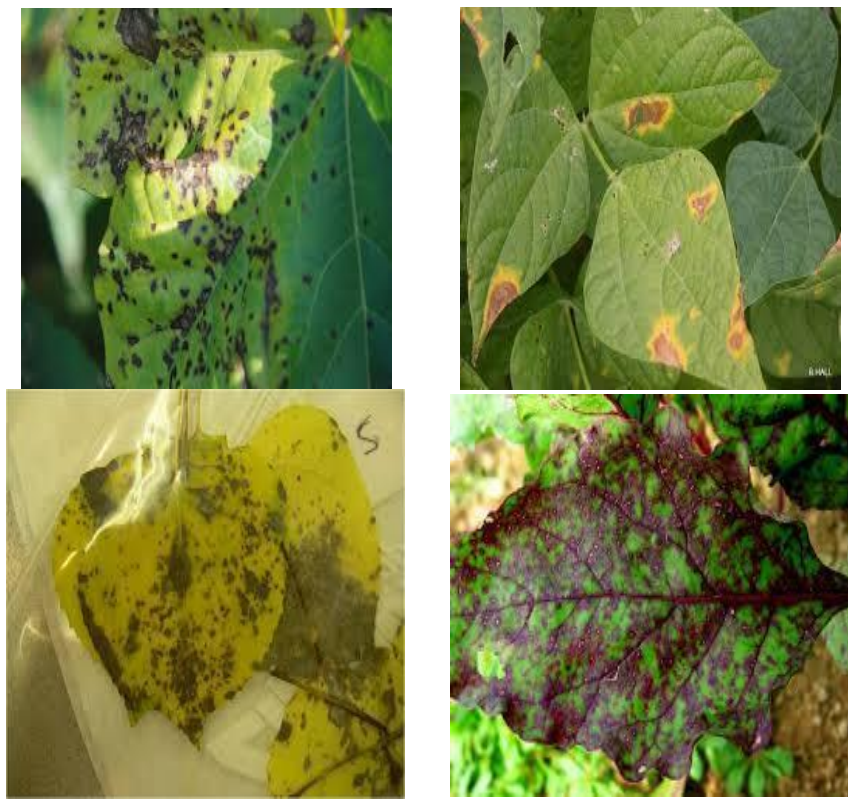

Step 4: In k-means clustering, classification is based on the minimizing the Euclidean Distance values which can be calculated using the Eq. 1:

$d(p, q)=d(q, p)=$

$\sqrt{\left(q_{1}-p_{1}\right)^{2}+\left(q_{2}-p_{2}\right)^{2}+\cdots+\left(q_{n}-p_{n}\right)^{2}}=$ $\sqrt{\sum_{i=1}^{n}\left(q_{i}-p_{i}\right)^{2}}$

Step 5: Based on the feature classes, image is segmented in three sub-feature images with three different type of Region of Interest (ROI).

Step 6: Select the Region of Interest from the segmented image.

Step 7: Convert the RGB color (ROI) image into grey scale image and maintain the Grey Level Occurrence Matrices (GLCMs).

Step 8: Extract the disease symptoms by calculating the feature values of Skewness, Standard_Deviation, Homogeneity, Contrast, Smoothness, Correlation, Kurtosis, Energy, Entropy, Mean, Variance, RMS, and IDM. These are calculated from disease affected portion.

Step 9: Apply SVM for the feature extraction and disease detection using the equation below:

$S V M=S V M t r a i n($ disease_feat, disease_type $)$

Step 1: Consider an image from the dataset of disease affected leaves.

Step 2: Pre-process the image by removing the noise and enhancing the contrast of the image using histogram equalization method. The intensity of the image is distributed using cumulative distribution function.

Step 3: Segment the enhanced image using kmeans clustering method. K-means clustering method clusters the image based in the presence of feature classes. where; SVMtrain is the SVM training function. disease_feat maintains the values of disease affected leaves of all the disease types. disease_type maintains the corresponding disease labels of Alternaria alternata, anthracnose, bacterial blight and Cercospora leaf spot.

Step 10: Apply the particle swarm optimization for the optimization and final classification.

10.1. All the image pixels are considered as the particles. Initially these particles randomly fly and search for the food sources in the form of 
affected disease portion in image. Then search for the local and global solution.

10.2. The performance of each particle depends upon the level of disease affected portion that has to optimize.

10.3. Each particle flies over the $n$ - dimensional outer search space and keep updating the following information:

- $\boldsymbol{X} \boldsymbol{i}$ - current position of particle

- $\boldsymbol{P i}$ - the personal best position of particle

- $\boldsymbol{V i}$ - the current velocity of particle

10.4. The velocity updates in PSO can be calculated using the formula given below by Eq. 3:

$V_{i^{(t+1)}}=\omega V_{i^{(t)}}+c_{1} r_{1}\left(P_{i^{(t)}}-X_{i^{(t)}}\right)+c_{2} r_{2}\left(P_{g}-X_{i^{(t)}}\right)$

Now, $\mathrm{V} i$ is the new velocity. So, the position of the particle updates with the velocity as below:

$\mathrm{X}_{\mathrm{i}}(\mathrm{t}+1)=\mathrm{X}_{\mathrm{i}}(\mathrm{t})+\mathrm{V}_{\mathrm{i}}(\mathrm{t}+1)$

10.5. Update the positions for each particle and store the global best solutions.

Step 11: Find the type of disease and evaluate the percentage of disease affected region by the ratio of disease data and leaf data.

Step 12: Compute the final accuracy of the concept with the maximum possible iterations.

\section{Result and discussion}

This section determines the evaluated results for the proposed integrated SVM \& PSO algorithm for plant leaf disease detection. Also the percentage of affected leaf portions determined with the overall accuracy of the concept.

\subsection{Results}

The proposed integrated concept of SVM \& PSO is implemented in MATLAB with GUI (Graphical User Interface). In this work, we are evaluating the results with the testing of whether the leaf is affected from the plant disease or not. If it found to be affected from the diseases, then further type/class of disease is evaluated with the percentage of affected leaf portion. Finally, results are evaluated based on the accuracy of the proposed concept.

In this process, image is initially considered in the form of RGB color value, then pre-processing and segmentation performed. During segmentation, we divide the image into three segments. Disease affected portion, unaffected portion and Partial affected portion. Further ROI is selected and proposed algorithm is performed to evaluate the type of disease and disease affected portion. Disease classes are Cercospora leaf spot, bacterial blight, anthracnose, and Alternaria alternata. Different leaves are affected with their different environmental effect and disease type. Accuracy of results varies from $95.16 \%$ to $98.38 \%$.

To check the performance of our proposed algorithm, we have considered the concepts of SVM and Improved SVM (Kaur and Kang, 2015).

The accuracy values for SVM and improved SVM with proposed concept are shown in Table 1.

Table 1: Performance comparison

\begin{tabular}{cc}
\hline Algorithm/Classifier & Accuracy Value (range in \%) \\
\hline SVM & $65-72 \%$ \\
Improved SVM & $69-79 \%$ \\
Integrated SVM and PSO & $95.16-98.38 \%$ \\
\hline
\end{tabular}

\section{Conclusion}

Human life is completely dependent upon nature and plants. So, there should be special methods to save plants from diseases. There is the existence of various traditional approaches to determine the plant diseases. Here, we have considered the integrated approach of SVM and nature inspired PSO method for plant leaf diseases detection. The dataset considered for the plant leaf is affected from the leaf diseases of Cercospora leaf spot, bacterial blight, anthracnose, and Alternaria alternata. We have evaluated the results to show the evaluated results and accuracy of the concept with the percentage of disease affected portion. Accuracy of the proposed integrated concept varies from $95.16 \%$ to $98.38 \%$. Also a performance comparison has been made to check the efficiency of proposed concept as compare to existing SVM and Improved SVM. So, we say that proposed integrated concept is efficient enough to determine the plant diseases.

\section{References}

Amari SI and Wu S (1999). Improving support vector machine classifiers by modifying kernel functions. Neural Networks, 12(6): 783-789.

Atas M, Yardimci Y, and Temizel A (2011). Aflatoxin contaminated chili pepper detection by hyperspectral imaging and machine learning. In the SPIE 8027 Conference on, Security, and Sensing, International Society for Optics and Photonics (SPIE), Bellingham, USA: 80270F-80270F. https:// doi.org/10.1117/12.883237

Barbedo JGA (2013). Digital image processing techniques for detecting, quantifying and classifying plant diseases. Springer Plus, 2(1):1-12.

Clerc M (2010). Particle swarm optimization. John Wiley and Sons, New Jersey, USA

Contreras-Medina LM, Osornio-Rios RA, Torres-Pacheco I, Romero-Troncoso RDJ, Guevara-González RG, and MillanAlmaraz JR (2012). Smart sensor for real-time quantification of common symptoms present in unhealthy plants. Sensors, 12(1): 784-805.

Dandawate Y and Kokare R (2015). An automated approach for classification of plant diseases towards development of futuristic decision support system in Indian perspective. In the International Conference on Advances in Computing, Communications and Informatics (ICACCI'15), IEEE, Kerala, India: 794-799. https://doi.org/10.1109/ICACCI.2015. 7275707

De Groot RS, Wilson MA, and Boumans RM (2002). A typology for the classification, description and valuation of ecosystem 
functions, goods and services. Ecological Economics, 41(3): 393-408.

Gavhale KR, Gawande U, and Hajari KO (2014). Unhealthy region of citrus leaf detection using image processing techniques. In the International Conference for Convergence of Technology, IEEE: 1-6. https://doi.org/10.1109/I2CT.2014.7092035

Kaur R and Kang SS (2015). An enhancement in classifier support vector machine to improve plant disease detection. In the IEEE $3^{\text {rd }}$ International Conference on MOOCs, Innovation and Technology in Education (MITE'15), IEEE, Amritsar, India: 135-140. https://doi.org/10.1109/MITE.2015.7375303

Kennedy J (2011). Particle swarm optimization. In: Sammut C and Webb GI (Eds.), Encyclopedia of Machine Learning: 760-766. Springer US, USA.

Khirade SD and Patil AB (2015). Plant disease detection using image processing. In the International Conference on Computing Communication Control and Automation (ICCUBEA'15), IEEE, Pune, India: 768-771. https://doi.org/10.1109/ICCUBEA.2015.153

Patil SB and Bodhe SK (2011). Leaf disease severity measurement using image processing. International Journal of Engineering and Technology, 3(5): 297-301.

Poli R, Kennedy J, and Blackwell T (2007). Particle swarm optimization. Swarm Intelligence, 1(1): 33-57.

Pujari JD, Yakkundimath R, and Byadgi AS (2014). December. Identification and classification of fungal disease affected on agriculture/horticulture crops using image processing techniques. In the IEEE International Conference on Computational Intelligence and Computing Research
(ICCIC'14), IEEE: 1-4. https://doi.org/10.1109/ICCIC.2014. 7238283

Raven PH, Evert RF, and Eichhorn SE (2005). Biology of plants. Macmillan, London, England.

Rothe PR and Kshirsagar RV (2015). Cotton leaf disease identification using pattern recognition techniques. In the International Conference on Pervasive Computing (ICPC'15), 2015, IEEE, Pune, India: 1-6. https://doi.org/10.1109/ PERVASIVE.2015.7086983

Sankaran S, Mishra A, Ehsani R, and Davis C (2010). A review of advanced techniques for detecting plant diseases. Computers and Electronics in Agriculture, 72(1): 1-13.

Singh V and Misra AK (2015). Detection of unhealthy region of plant leaves using Image Processing and Genetic Algorithm. In the International Conference on Advances in Computer Engineering and Applications (ICACEA'15), IEEE: 1028-1032. https://doi.org/10.1109/ICACEA.2015.7164858

Suykens JA and Vandewalle J (1999). Least squares support vector machine classifiers. Neural Processing Letters, 9(3): 293-300.

Tong S and Chang E (2001). Support vector machine active learning for image retrieval. In the $9^{\text {th }}$ ACM International Conference on Multimedia, ACM, Ottawa, Canada: 107-118. https://doi.org/10.1145/500141.500159

Wang H, Li G, Ma Z, and Li X (2012). Application of neural networks to image recognition of plant diseases. In the International Conference on Systems and Informatics (ICSAI'12), IEEE, Yantai, China: 2159-2164. https://doi.org/10.1109/ICSAI.2012.6223479 